\title{
Thermal Correction to the Kinnersley Black Hole in a Lorentz-Violating Dirac Field Theory
}

\author{
Zhi-E. Liu ${ }^{1 *}$, Jie Zhang ${ }^{1}$ and Shu-Zheng Yang ${ }^{2}$ \\ ${ }^{1}$ College of Physics and Electronic Engineering, Qilu Normal University, Jinan, China, ${ }^{2}$ Physics and Astronomy College, China \\ West Normal University, Nanchong, China
}

According to Lorentz-violating theory, the dynamical equation of Dirac particles in the Kinnersley black hole with variably accelerated linear motion is modified. The Hawking quantum tunneling radiation characteristics of Kinnersley black hole are obtained by solving the modified equation. The expression of the Hawking temperature of Kinnersley black hole has been updated.

Keywords: Kinnersley black hole, quantum tunneling radiation, Hawking temperature, Lorentz symmetry violating, Dirac particle

OPEN ACCESS

Edited by:

Mohamed Chabab,

Cadi Ayyad University, Morocco

Reviewed by:

Izzet Sakalli,

Eastern Mediterranean University,

Turkey

Adil Belhaj,

Mohammed V University, Morocco

${ }^{*}$ Correspondence:

Zhi-E. Liu

zhieliu@163.com

Specialty section:

This article was submitted to

High-Energy and Astroparticle

Physics,

a section of the journal

Frontiers in Physics

Received: 21 August 2021 Accepted: 28 October 2021

Published: 29 November 2021

Citation:

Liu Z-E, Zhang J and Yang S-Z (2021) Thermal Correction to the Kinnersley Black Hole in a Lorentz-Violating Dirac

Field Theory.

Front. Phys. 9:762279.

doi: 10.3389/fphy.2021.762279

\section{INTRODUCTION}

During past years, people have made many meaningful researches on the quantum tunneling radiation of black holes [1-15]. These researches have enriched us with knowledge about the thermodynamic evolution of black holes. Using the Hawking quantum tunneling radiation theory, Kraws et al calculated the Hawking temperature and entropy of black hole. The research of quantum tunneling radiation for black hole has been increased greatly by the semiclassical Hamilton-Jacobi method $[8,9]$. Kerner and Mann used the semiclassical theory to study the quantum tunneling radiation characteristic of Dirac field particles in black hole [16, 17]. They divided the fermion spin into up and down and decomposed the Dirac equations into two groups, then obtained the tunneling rate of Dirac particles at the event horizon and the Hawking temperature of black hole.

In 2009, Lin and Yang proposed a new method to study the quantum tunneling radiation of black hole. They transformed the Dirac equation in curved space-time into a matrix equation by using the semiclassical approximation theory, and then the resulting matrix equation was further converted to the Hamilton-Jacobi equation for Dirac particles in curved space-time by using the commutation relation of gamma matrices. Finally they derived the Hawking quantum tunneling rate of fermions and other important physical quantities of black hole based on the Hamilton-Jacobi equation [11, 14, 18-21]. Their work showed that the Hamilton-Jacobi equation and its Hamilton principal function $S$ can be applied to the study of quantum tunneling radiation of fermions in curved space-time. The developing Hamilton-Jacobi method can effectively solve the problems related to fermion tunneling radiation and unify the expressions of quantum tunneling radiation theory in curved space-time.

The study on modifications to the Hawking radiation of black holes can help for understanding the information loss paradox [22-26]. Banerjee and Majhi extended the beyond semiclassical approximation to include all quantum corrections [27-32]. Lin and Yang investigate tunnelling of charged black holes based on Klein-Gordon scalar particle theory to derive corrections to the tunnelling rate and temperature in Reissner-Nordström space-time and Reissner Nerdström-de Sitter space-time, respectively [33]. Beyond semiclassical theory and semiclassical theory are 
both modified theories, for which the former can give more accurate correction to the quantum tunneling rate of black hole.

With research of string theory and quantum gravity theory, people have realized that the Lorentz relation should be modified at high energy, which will lead to the Dirac equation in curved space-time to be modified. The application of Lorentz-violating theory in curved space-time is a Frontier subject worthy of attention [34-40]. Cruz et al introduced the Lorentz symmetry violating term in the scalar field Lagrangian, namely the Lorentzviolating scalar field theory, to research thermal corrections to the Casimir energy [41]. The Dirac particle action and Dirac equation with Lorentz symmetry violating in flat space-time are introduced in [42]. The influence of Lorentz violating theory on the quantum tunneling radiation of Dirac particles in the Vaidya black hole is investigated in [43].

However, for the stationary and dynamic axisymmetric black holes, the effect of Lorentz violating theory on their quantum tunneling radiation has not been studied. In this paper, Lorentz violating theory is considered to correct the quantum tunneling rate, the temperature and entropy of a dynamic Kinnersley black hole with variably accelerated linear motion, resulting in some new conclusion.

In the second section, we introduce the modification of Lorentz violating Dirac field theory to Dirac particle dynamics equation in the Kinnersley space-time. The third section studies the thermal and entropy corrections to Kinnersley black hole in a Lorentz violating Dirac field theory. The last section gives a discussion on the results obtained in this paper.

\section{LORENTZ'S VIOLATING THEORY AND DIRAC EQUATION IN DYNAMICAL CURVED SPACE-TIME}

According to Hamilton principle, the dynamic equation of Dirac particle in flat space-time can be acquired from the action of Dirac particle in flat space-time [41-43]. Adding the Lorentz symmetry violating term into the action and using Hamilton principle, we can obtain the Dirac equation of Lorentz symmetry violating in a flat space-time. It only needs to pay attention to two points for generalizing the particle dynamics equation from the flat space-time to the curved space-time: one is to generalize the gamma matrix $\gamma^{\mu}$ from the flat space-time to the curved spacetime, with different curved space-time having different $\gamma^{\mu}$; the other is to generalize the ordinary derivative to the covariant derivative related to connection. Therefore, in the Kinnersley curved space-time, the dynamics equation of spin $1 / 2$ fermion with Lorentz symmetry violating can be expressed as [42].

$$
\begin{gathered}
\left\{\gamma^{\mu} D_{\mu}\left[1+\hbar^{2} \frac{a}{m^{2}}\left(\gamma^{\mu} D_{\mu}\right)^{2}\right]+\frac{b}{\hbar} \gamma^{5}+c \hbar\left(u^{\alpha} D_{\alpha}\right)^{2}\right. \\
\left.-\frac{m}{\hbar}\right\} \Psi=0
\end{gathered}
$$

where $\Psi$ is the wave function. For Dirac particles, the wave function $\Psi$ and the action $S$ are linked by

$$
\Psi=\psi_{0} e^{\frac{i}{h} S}
$$

where $\psi_{0}$ is a column matrix. For Dirac particles the nonstationary Kinnersley black hole

$$
S=S(\nu, r, \theta, \phi),
$$

where $v$ is the advanced Eddington coordinate. The covariant derivative $D_{\mu}$ in Eq. 1 is defined by

$$
D_{\mu}=\partial_{\mu}+\frac{\mathrm{i}}{2} \Gamma_{\mu}^{\alpha \beta} \Pi_{\alpha \beta},
$$

where $\Gamma_{\mu}^{\alpha \beta}$ is the connection in Riemannian geometry, and $\Pi_{\alpha \beta}$ is expressed as

$$
\Pi_{\alpha \beta}=\frac{\mathrm{i}}{4}\left[\gamma^{\alpha}, \gamma^{\beta}\right]
$$

$\frac{\mathrm{i}}{\hbar} \Gamma_{\mu}^{\alpha \beta} \Pi_{\alpha \beta}$ is the spin connection term that characterizes the spinor covariant derivative in curved space-time. In Eq.1, $a, b$ and $c$ are all small quantities that satisfy $a, b, c \ll m$, where $m$ is particle mass. The Gamma matrices $\gamma^{\mu}$ or $\gamma^{\nu}$ meet the following anticommutation relation:

$$
\begin{gathered}
\gamma^{\mu} \gamma^{\nu}+\gamma^{\nu} \gamma^{\mu}=2 g^{\mu \nu} I, \\
\gamma^{5} \gamma^{\mu}+\gamma^{\mu} \gamma^{5}=0 .
\end{gathered}
$$

The $4 \mathrm{D}$ ether-like field vector $u^{\alpha}$ is not a constant vector in curved space-time, but it must satisfy the following condition

$$
u^{\alpha} u_{\alpha}=\mathrm{c}(\text { constant })
$$

We substitute Eq. 2 into Eq. 1, and keep the terms associated with the lowest order of $\hbar$, then Eq. 1 is reduced to

$$
\begin{gathered}
\left\{\mathrm{i} \gamma^{\mu} \partial_{\mu} S\left[1-\frac{a}{m^{2}} \gamma^{\alpha} \gamma^{\beta} \partial_{\alpha} S \partial_{\beta} S\right]-c u^{\alpha} u^{\beta} \partial_{\alpha} S \partial_{\beta} S\right. \\
\left.+b \gamma^{5}-m\right\} \Psi=0 .
\end{gathered}
$$

Considering Eq. 6, the following equality holds:

$$
\gamma^{\alpha} \gamma^{\beta} \partial_{\alpha} S \partial_{\beta} S=g^{\alpha \beta} \partial_{\alpha} S \partial_{\beta} S .
$$

Then the dynamics Eq. 9 becomes

$$
\begin{gathered}
\mathrm{i} \gamma^{\mu} \partial_{\mu} S \Psi=\left(1-\frac{a}{m^{2}} g^{\alpha \beta} \partial_{\alpha} S \partial_{\beta} S\right)^{-1} \\
\left(c u^{\alpha} u^{\alpha} \partial_{\alpha} S \partial_{\beta} S-b \gamma^{5}+m\right) \Psi \\
\approx\left[1+\left(\frac{c}{m} u^{\alpha} u^{\beta}+\frac{a}{m^{2}} g^{\alpha \beta}\right) \partial_{\alpha} S \partial_{\beta} S-\frac{b}{m} \gamma^{5}\right] m \Psi,
\end{gathered}
$$

Taking square for both sides of Eq. 11, and omitting the $\frac{b}{m} \gamma^{5}$ term, we transform it into

$$
\begin{aligned}
& -\gamma^{\mu} \gamma^{\nu} \partial_{\mu} S \partial_{\gamma} S \Psi \\
& =m^{2} \psi+2\left(c m u^{\alpha} u^{\beta}+a g^{\alpha \beta}\right) \partial_{\alpha} S \partial_{\beta} S \Psi+\mathcal{O}
\end{aligned}
$$

where $\mathcal{O}$ is a high order small quantity. Then we use Eq. 6 to simplify Eq. 12, resulting in

$$
\left[g^{\mu \nu} \partial_{\mu} S \partial_{\nu} S+2\left(c m u^{\mu} u^{\nu}+a g^{\mu \nu}\right) \partial_{\mu} S \partial_{\nu} S+m^{2}\right] \Psi=0
$$


Obviously, $\Psi \neq 0$, making it necessary that

$$
g^{\mu \nu} \partial_{\mu} S \partial_{\nu} S+2\left(c m u^{\mu} u^{\nu}+a g^{\mu \nu}\right) \partial_{\mu} S \partial_{\nu} S+m^{2}=0 .
$$

In this equation $S$ is the action, also called Hamiltonian principal function. In the process of derive this equation, we ignore the term $\frac{b}{m} \gamma^{5}$ for two reasons. One is that $b$ is a small quantity; the other is that the term $\frac{b}{m} \gamma^{5}$ in the square bracket of Eq. 11 only indicates the correction in quantity and thus can be ignored. However, the terms other than $\frac{b}{m} \gamma^{5}$ in the square bracket of Eq. 11 are related to the metric tensor or ether-like field vector, thus can not be ignored. In fact, Eq. 14 is completely equivalent to Eq. 1, since Eq. 14 is also the dynamic equation of Dirac particles. Eq. 14 is a new form of modified Hamilton-Jacobi equation about the action $S$ of Dirac particles. Starting from Eq. 14, we can conveniently study the tunneling radiation characteristics of fermion with mass $m$ in the curved non-stationary Kinnersley black hole. This is an innovation that has not been reported yet.

For a Dirac particle with mass of $m$ and charge $q$, its dynamic equation is very complicated. Firstly, Eq. 4 must be modified to

$$
D_{\mu}=\partial_{\mu}-\frac{\mathrm{i}}{\hbar} q A_{\mu}+\frac{\mathrm{i}}{2} \Gamma_{\mu}^{\alpha \beta} \Pi_{\alpha \beta} .
$$

Substituting Eq. 15 into Eq. 1 and using the same method as deriving Eq. 14, we can get the dynamic equation of Dirac particle with mass $m$ and charge $q$ as follows

$$
\begin{aligned}
& {\left[g^{\mu \nu}(1+2 a)+2 c m u^{\mu} u^{\nu}\right]\left(\partial_{\mu} S-q A_{\mu}\right)\left(\partial_{\nu} S-q A_{\nu}\right)} \\
& +m^{2}=0 .
\end{aligned}
$$

During the derivation of Eqs 14, 16, we ignored higher order quantities in terms of $a$ and $c$. From the point of view of mathematics and physics, this is a reasonable and effective approximation. In the next section, we will study the tunneling radiation of Dirac particles in the non-stationary Kinnersley black hole according to Eq. 16.

\section{THERMAL CORRECTION TO THE KINNERSLEY BLACK HOLE IN THE LORENTZ-VIOLATING THEORY}

According to Kinnersley's research on the metric of accelerating black holes, the space-time line element of a linearly moving black hole with variable acceleration described by the advanced Eddington coordinate $v$ is [44].

$$
\begin{gathered}
\mathrm{d} s^{2}=\left(1-2 a_{k} r \cos \theta-r^{2} f^{2}-2 M r^{-1}\right) \mathrm{d} v^{2}-2 \mathrm{~d} v \mathrm{~d} r \\
-2 r^{2} f \mathrm{~d} v \mathrm{~d} \theta-r^{2} \mathrm{~d} \theta^{2}-r^{2} \sin ^{2} \theta \mathrm{d} \phi^{2}, \\
f=-a_{k} \sin \theta,
\end{gathered}
$$

where $a_{k}=a_{k}(v)$ is the acceleration of the Kinnersley black hole, $\theta$ and $\phi$ are spherical coordinates, and $M$ is the mass of the black hole. The north pole $\theta=0$ of the black hole always points in the direction of acceleration. Therefore, the covariant and contravariant metric tensors are

$$
\begin{aligned}
g_{\mu \nu} & =\left(\begin{array}{cccc}
g_{00} & -1 & -r^{2} f & 0 \\
-1 & 0 & 0 & 0 \\
-r^{2} f & 0 & -r^{2} & 0 \\
0 & 0 & 0 & -r^{2} \sin ^{2} \theta
\end{array}\right) \\
g^{\mu \nu} & =\left(\begin{array}{cccc}
0 & -1 & 0 & 0 \\
-1 & g^{11} & f & 0 \\
0 & f & -r^{-2} & 0 \\
0 & 0 & 0 & -r^{-2} \sin ^{-2} \theta
\end{array}\right)
\end{aligned}
$$

where

$$
\begin{gathered}
g 00=1-2 a_{k} r \cos \theta-r^{2} f^{2}-2 M r^{-1} \\
g^{11}=-\left(1-2 a_{k} r \cos \theta-2 M r^{-1}\right)
\end{gathered}
$$

Obviously, this black hole is uncharged. According to the zero hypersurface equation

$$
g^{\mu \nu} \frac{\partial F}{\partial x^{\mu}} \frac{\partial F}{\partial x^{\nu}}=0
$$

The event horizon of the black hole should satisfy the following equation:

$$
2 \dot{r}_{H}-\left(1-2 a_{k} r \cos \theta-2 M r^{-1}\right)-2 f r_{H}^{\prime}-\left(\frac{r_{H}^{\prime}}{r}\right)^{2}=0,
$$

where $\dot{r}_{H}=\frac{\partial r_{H}}{\partial v}, r_{H}^{\prime}=\frac{\partial r_{H}}{\partial \theta}$. As one of fundamental characteristics, the Kinnersley black hole is axial symmetric and has Killing vector $\left(\frac{\partial}{\partial \phi}\right)^{\alpha}$. Now, by using Eqs 16, 20 is reduced to

$$
\begin{aligned}
& (1+2 a)\left[g^{11}\left(\partial_{r} S-q A_{r}\right)^{2}-2\left(\partial_{r} S-q A_{r}\right)\left(\partial_{v} S-q A_{v}\right)\right. \\
& +2 f\left(\partial_{r} S-q A_{r}\right)\left(\partial_{\theta} S-q A_{\theta}\right)-\frac{1}{r^{2}}\left(\partial_{\theta} S-q A_{\theta}\right)^{2} \\
& \left.-\frac{1}{r^{2} \sin ^{2} \theta}\left(\partial_{\phi} S-q A_{\phi}\right)^{2}\right] \\
& +2 c m u^{u} u^{\nu}\left(\partial_{\mu} S-q A_{\mu}\right)\left(\partial_{\nu} S-q A_{\nu}\right)+m^{2}=0 .
\end{aligned}
$$

Note that $A_{\mu}$ is not the electromagnetic potential of the Kinnersley black hole itself, but the electromagnetic potential of the cosmological space around the moving non-charged Kinnersley black hole. According to Eqs 17, 19, 20, we construct the ether-like field vector $u^{\mu}$ such that

$$
u^{v}=\frac{c_{v}}{\sqrt{g_{00}}}, u^{r}=\frac{c_{r}}{g_{01}} \sqrt{g_{00}}, u^{\theta}=\frac{c_{\theta}}{g_{02}} \sqrt{g_{00}}, u^{\phi}=\frac{c_{\phi}}{\sqrt{g_{33}}}
$$

where $c_{v}, c_{r}, c_{\theta}, c_{\phi}$ are all constants. It is easy to show that $u^{v} u_{v}=c_{v}^{2}, u^{r} u_{r}=c_{v} c_{r}, u^{\theta} u_{\theta}=c_{v} c_{\theta}, u^{\phi} u_{\phi}=c_{\phi}^{2}$ and thus condition (8) is met. Substituting Eq. 26 into Eq. 25 will result in 


$$
\begin{aligned}
& (1+2 a)\left[g^{11}\left(\partial_{r} S-q A_{r}\right)^{2}-2\left(\partial_{r} S-q A_{r}\right)\left(\partial_{v} S-q A_{v}\right)\right. \\
& +2 f\left(\partial_{r} S-q A_{r}\right)\left(\partial_{\theta} S-q A_{\theta}\right)-\frac{1}{r^{2}}\left(\partial_{\theta} S-q A_{\theta}\right)^{2} \\
& \left.-\frac{1}{r^{2} \sin ^{2} \theta}\left(\partial_{\phi} S-q A_{\phi}\right)^{2}\right]+2 c m\left[\frac{c_{v}^{2}}{g_{00}}\left(\partial_{v} S-q A_{v}\right)^{2}\right. \\
& -2 c_{v} c_{r}\left(\partial_{r} S-q A_{r}\right)\left(\partial_{v} S-q A_{v}\right) \\
& -2 c_{v} c_{\theta} r^{-2} f^{-1}\left(\partial_{v} S-q A_{v}\right)\left(\partial_{\theta} S-q A_{\theta}\right) \\
& +2 c_{v} c_{\phi}\left(g_{00} g_{33}\right)^{-\frac{1}{2}}\left(\partial_{v} S-q A_{v}\right)\left(\partial_{\phi} S-q A_{\phi}\right) \\
& +c_{r}^{2} g_{00}\left(\partial_{r} S-q A_{r}\right)^{2} \\
& +2 c_{r} c_{\theta} g_{00} r^{-2} f^{-1}\left(\partial_{r} S-q A_{r}\right)\left(\partial_{\theta} S-q A_{\theta}\right) \\
& \quad \frac{1}{2} \quad-\frac{1}{2}\left(\partial_{r} S-q A_{r}\right)\left(\partial_{\phi} S-q A_{\phi}\right) \\
& +c_{r} c_{\phi} g_{00}^{2} g_{33}^{2} g_{00} r^{-4} f^{-2}\left(\partial_{\theta} S-q A_{\theta}\right)^{2} \\
& \quad \frac{1}{2} \quad-\frac{1}{2} \\
& -2 c_{\theta} c_{\phi} g_{00} g_{33} r^{-2} f^{-1}\left(\partial_{\theta} S-q A_{\theta}\right)\left(\partial_{\phi} S-q A_{\phi}\right) \\
& \left.+c_{\phi}^{2} g_{33}^{-1}\left(\partial_{\phi} S-q A_{\phi}\right)^{2}\right]+m^{2}=0 .
\end{aligned}
$$

This is a time-dependent equation. To solve this equation, general tortoise coordinate transformation must be performed, i.e.

$$
\begin{aligned}
& r_{*}=r+\frac{1}{2 \kappa\left(v_{0}, \theta_{0}\right)} \ln \frac{r-r_{H}(v, \theta)}{r_{H}\left(v_{0}, \theta_{0}\right)}, \\
& v_{*}=v-v_{0}, \\
& \theta_{*}=\theta-\theta_{0} .
\end{aligned}
$$

Therefore, we have

$$
\begin{gathered}
\frac{\partial}{\partial r}=\frac{1+2 \kappa\left(r-r_{H}\right)}{2 \kappa\left(r-r_{H}\right)} \frac{\partial}{\partial r_{*}}, \\
\frac{\partial}{\partial \theta}=\frac{\partial}{\partial \theta_{*}}-\frac{r_{H}^{\prime}}{2 \kappa\left(r-r_{H}\right)} \frac{\partial}{\partial r_{*}}, \\
\frac{\partial}{\partial \nu}=\frac{\partial}{\partial v_{*}}-\frac{\dot{r}_{H}}{2 \kappa\left(r-r_{H}\right)} \frac{\partial}{\partial r_{*}} .
\end{gathered}
$$

Then, the following variable separation is performed for $S$

$$
S=R(v, r, \theta)+j \phi
$$

and let

$$
\begin{gathered}
\frac{\partial S}{\partial \nu_{*}}=-\omega, \\
\frac{\partial S}{\partial \phi}=j, \\
\frac{\partial S}{\partial \theta_{*}}=p_{\theta} .
\end{gathered}
$$

where $\omega$ is the particle energy, $p_{\theta}$ is a component of the particle generalized momentum in the $\theta$ direction, and the constant $j$ is a component of the particle generalized moment in the $\phi$ direction. Substituting Eqs 29-31, 33-35 into Eq. 27, and considering the special condition of $r \rightarrow r_{H}, \theta \rightarrow \theta_{0}, v \rightarrow v_{0}$, we can get the dynamic equation of Dirac particles at the event horizon of the black hole as follows

$$
\lim _{\substack{r \rightarrow r_{H} \\ v \rightarrow v_{0} \\ \theta \rightarrow \theta_{0}}} \frac{A}{B}\left(\frac{\partial S}{\partial r_{*}}\right)^{2}-2 \omega \frac{\partial S}{\partial r_{*}}+2 \lim _{\substack{r \rightarrow r_{H} \\ v \rightarrow v_{0} \\ \theta \rightarrow \theta_{0}}} \frac{C}{B} \frac{\partial S}{\partial r_{*}}=0,
$$

where

$$
\begin{aligned}
& A= \\
& {\left[2 \kappa\left(r-r_{H}\right)\right]^{-1}\left\{(1+2 a)\left[g^{11}+2 \dot{r}_{H}-2 f r_{H}^{\prime}-\left(\frac{r_{H}^{\prime}}{r}\right)^{2}\right]\right\}} \\
& -2 c m\left[\dot{r}_{H}^{2} c_{v}^{2} g_{00}^{-1}+2 c_{v} c_{r} \dot{r}_{H}-2 c_{v} c_{\theta} r^{-2} f^{-1} \dot{r}_{H} r_{H}^{\prime}\right. \\
& \left.+c_{r}^{2} g_{00}+2 c_{v} c_{\theta} r^{-2} f^{-1} g_{00} r_{H}^{\prime}+c_{\theta}^{2} g_{00} r_{H}^{\prime}\right] \text {, } \\
& \left.B\right|_{r \rightarrow r_{H}}=B^{\prime}=(1+2 a) \\
& \begin{array}{l}
v \rightarrow v_{0} \\
\theta \rightarrow \theta_{0}
\end{array} \\
& +2 c m\left[c _ { v } ^ { 2 } \dot { r } _ { H } \left(1-2 a_{k} \cos \theta_{0}-r_{H}^{2} a_{k}^{2} \sin ^{2} \theta_{0}\right.\right. \\
& \left.\left.-2 M r_{H}^{-1}\right)^{-1}+c_{v} c_{r}+c_{v} c_{\theta} r_{H}^{-2} a_{k}^{-1} \sin ^{-1} \theta_{0} r_{H}^{\prime}\right] \text {, } \\
& \left.C\right|_{r \rightarrow r_{H}}=C^{\prime}=(1+2 a) q\left\{A_{0}+A_{1}\left[1-2 a_{k} r_{H} \cos \theta_{0}\right.\right. \\
& \begin{array}{l}
v \rightarrow v_{0} \\
\theta \rightarrow \theta_{0}
\end{array} \\
& \left.\left.-2 M r_{H}^{-1}-\dot{r}_{H}+f r_{H}^{\prime}\right]-A_{2}\left(r_{H}^{\prime} r^{-2}-a_{k} \sin \theta_{0}\right)\right\} \\
& +(1+2 a) p_{\theta}\left(r_{H}^{-2} r_{H}^{\prime}-a_{k} \sin \theta_{0}\right)+2 c m\left\{q A _ { 0 } c _ { v } \left[c_{r}\right.\right. \\
& +c_{v} \dot{r_{H}}\left(1-2 a_{k} \cos \theta_{0}-r_{H}^{2} a_{k}^{2} \sin ^{2} \theta_{0}-2 M r_{H}^{-1}\right)^{-1} \\
& \left.+c_{\theta} r_{H}^{-2} a_{k}^{-1} \sin ^{-1} \theta_{0} r_{H}^{\prime}\right]-c_{r} q A_{1}\left[c_{v} \dot{r}_{H}+(1\right. \\
& \left.-2 a_{k} r_{H} \cos \theta_{0}-r_{H}^{2} a_{k}^{2} \sin ^{2} \theta_{0}-2 M r_{H}^{-1}\right) c_{r} \\
& \left.\left.-c_{\theta} r_{H}^{-2} a_{k}^{-1} \sin ^{-1} \theta_{0} r_{H}^{\prime}\right)\right]+c_{\theta} q A_{2} r_{H}^{-2} a^{-1} \sin ^{-1} \theta_{0} \\
& \left(1-2 a_{k} r_{H} \cos \theta_{0}-r_{H}^{2} a_{k}^{2} \sin \theta_{0}-2 M r_{H}^{-1}\right) \\
& \left(c_{\theta} r_{H}^{-2} r_{H}^{\prime} a_{k}^{-1} \sin ^{-1} \theta_{0}+c_{r}\right)+q c_{\phi} A_{3} r_{H}^{-1} \sin ^{-1} \theta_{0} \\
& {\left[-c_{v} \dot{r}_{H}\left(2 a_{k} r_{H} \cos \theta_{0}-1+r_{H}^{2} a_{k}^{2} \sin ^{2} \theta_{0}+2 M r_{H}^{-1}\right)^{-\frac{1}{2}}\right.} \\
& a_{k}^{-1} \sin ^{-1} \theta_{0}+\left(2 a_{k} r_{H} \cos \theta_{0}-1+r_{H}^{2} a_{k}^{2} \sin ^{2} \theta_{0}\right. \\
& \left.\left.\left.+2 M r_{H}^{-1}\right)^{\frac{1}{2}}\left(c_{r}+c_{\theta} r_{H}^{-2} a_{k}^{-1} \sin ^{-1} \theta_{0}\right)\right]+C^{\prime \prime}\right\} \text {, }
\end{aligned}
$$

and

$$
\begin{aligned}
& C^{\prime \prime}=-c_{v} c_{\theta} r_{H}^{-2} \dot{r}_{H} p_{\theta} a_{k}^{-1} \sin ^{-1} \theta_{0}+c_{v} c_{\phi} \dot{r}_{H} j \\
& \left(2 a_{k} r_{H} \cos \theta-1+r_{H}^{2} a_{k}^{2} \sin ^{2} \theta_{0}+2 M r_{H}^{-1}\right)^{-\frac{1}{2}} r_{H}^{-1} a_{k}^{-1} \sin ^{-2} \theta_{0} \\
& -c_{r} c_{\theta} p_{\theta} r_{H}^{-2} a_{k}^{-1} \sin ^{-1} \theta_{0}\left(1-2 a_{k} r_{H} \cos \theta-r_{H}^{2} f^{2}-2 M r_{H}^{-1}\right) \\
& +c_{\phi}\left(2 a_{k} r_{H} \cos \theta-1+r_{H}^{2} a_{k}^{2} \sin ^{2} \theta_{0}+2 M r_{H}^{-1}\right)^{\frac{1}{2}} \\
& r^{-1} \sin ^{-1} \theta_{0}\left(c_{r} j-c_{\theta} r^{-2} a_{k}^{-1} \sin ^{-1} \theta_{0} r_{H}^{\prime} j\right) \\
& -c_{\theta}^{2} r_{H}^{-4} a_{k}^{-2} \sin ^{-2} \theta_{0} p_{\theta} r_{H}^{\prime}\left(1-2 a_{k} r_{H} \cos \theta\right. \\
& \left.-r_{H}^{2} a_{k}^{2} \sin ^{2} \theta-2 M r_{H}^{\prime}\right) .
\end{aligned}
$$


Let

$$
\lim _{\substack{r \rightarrow r_{H} \\ v \rightarrow v_{0} \\ \theta \rightarrow \theta_{0}}} \frac{A}{B}=1 .
$$

The first part of the expression (37) of $A$ will become an indeterminate formula of type $\frac{0}{0}$ as $r \rightarrow r_{H}, v \rightarrow v_{0}, \theta \rightarrow \theta_{0}$. So substituting Eqs 37, 38, into Eq. 41 and using L'Hospital rule, we get

$$
\begin{gathered}
\kappa\left(v_{0}, \theta_{0}\right)=\kappa \\
=\frac{\frac{M}{r_{H}^{2}}+a_{k} \cos \theta_{0}-\frac{\left(r_{H}^{\prime}\right)^{2}}{r_{H}^{3}}}{(1+2 a)+2 c m\left(\dot{r}_{H}+c_{v} c_{r}+2 c_{v} c_{\theta} r_{H}^{2} a_{k}^{-1} \sin ^{-1} \theta_{0}\right)}
\end{gathered}
$$

where $a_{k}=a_{k}\left(v_{0}\right) . \kappa\left(v_{0}, \theta_{0}\right)$ in Eqs 29-31 is directly related to the small region $v_{0}, \theta_{0}$ at the event horizon $r_{H}$ of the black hole. In fact $\kappa$ is the surface gravity of the black hole. Let

$$
\omega_{0}=\lim _{\substack{r \rightarrow r_{H} \\ v \rightarrow v_{0} \\ \theta \rightarrow \theta_{0}}} \frac{C^{\prime}}{B^{\prime}},
$$

Be the chemical potential, also known as the maximal interleaving of Dirac energy levels of particles. Then Eq. 36 is reduced to

$$
\left(\frac{\partial S}{\partial r_{*}}\right)^{2}-2\left(\omega-\omega_{0}\right) \frac{\partial S}{\partial r_{*}}=0 .
$$

By solving this equation, we get

$$
\frac{\partial S}{\partial r_{*}}=\left(\omega-\omega_{0}\right) \pm\left(\omega-\omega_{0}\right) .
$$

According to Eq. 29, we have

$$
\frac{\partial S}{\partial r}=\frac{1+2 \kappa\left(r-r_{H}\right)}{2 \kappa\left(r-r_{H}\right)} \frac{\partial S}{\partial r_{*}}
$$

Therefore, the action $S$ of Dirac particle with mass $m$ and charge $Q$ can be obtained by integrating this equation and using the residue theorem, that is

$$
S_{ \pm}=\frac{\mathrm{i} \pi}{2 \kappa}\left[\left(\omega-\omega_{0}\right) \pm\left(\omega-\omega_{0}\right)\right]
$$

According to the quantum tunneling radiation theory and the semiclassical WKB approximation theory, the tunneling rate of Dirac particles at the event horizon of the non-stationary kennersley black hole should be

$$
\Gamma \sim \exp \left(-2 \operatorname{Im}\left(S_{+}-S_{-}\right)=\exp \left(-\frac{\omega-\omega_{0}}{T_{H}}\right) .\right.
$$

Here, $T_{H}$ is the Hawking temperature at the event horizon of the non-stationary Kennersley black hole, corrected by the Lorentz symmetry violating theory. It is linked to the surface gravity $\kappa$ at the event horizon by $T_{H}=\frac{\kappa}{2 \pi}$. From Eq. 42, we get

$$
\begin{aligned}
& T_{H}= \\
& \frac{M+a_{k} r_{H}^{2} \cos \theta_{0}-\left(r_{H}^{\prime}\right)^{2} r_{H}^{-1}}{2 \pi r_{H}^{2}\left[1+2 a+2 c m\left(\dot{r}_{H}+c_{v} c_{r}+2 c m c_{v} c_{\theta} a_{k}^{-1} \sin ^{-1} \theta_{0}\right)\right]} \\
& =T_{h}\left[1-2\left(a+c m \dot{r}_{H}+c m c_{v} c_{r}\right.\right. \\
& \left.\left.+2 c m c_{v} c_{\theta} a_{k}^{-1} \sin ^{-1} \theta_{0} r_{H}^{-2}\right)+\cdots\right],
\end{aligned}
$$

where

$$
T_{h}=\frac{M+a_{k} r_{H}^{2} \cos \theta_{0}-\left(r_{H}^{\prime}\right)^{2} r_{H}^{-1}}{2 \pi r_{H}^{2}}
$$

is the uncorrected Hawking temperature at the event horizon of the black hole. For clarity, only the terms of the zeroth and first order are showed in Eq. 49. It can be seen from Eq. 49 that the coefficients $a, c$ of the correction term in Eq. 1 and the components $u^{v}, u^{r}, u^{\theta}$ of ether-like field vector all have an effect on $T_{H}$. Since there is killing vector $\left(\frac{\partial}{\partial \phi}\right)^{\alpha}$ in this spacetime, $u^{\phi}$ has no effect on $T_{H}$. Moreover, according to Eqs 38-40, 43, 48, the tunneling rate $\Gamma$ and chemical potential $\omega_{0}$ of Dirac particles in this space-time are also corrected, and similarly, the quantities $a, c, u^{v}, u^{r}, u^{\theta}, u^{\phi}$ all have influence on $\Gamma$ and $\omega_{0}$. What needs to be further explained is that $A_{0}, A_{1}, A_{2}$, and $A_{3}$ in Eqs 39, 40 in fact correspond to $A_{v}, A_{r}, A_{\theta}$ and $A_{\phi}$ in Eq. 27. The only difference is that $r$ and $\theta$ have been replaced by $r_{H}$ and $\theta_{0}$ in $A_{0}, A_{1}, A_{2}$, and $A_{3}$. Obviously, $A_{\mu}$ has also an effect on $\Gamma$ and $\omega_{0}$. If $A_{\mu}=0$, then

$$
C^{\prime}=C_{1}^{\prime}=(1+2 a) p_{\theta}\left(r_{H}^{2} r_{H}^{\prime}-a_{k} \sin \theta_{0}\right)+2 c m C^{\prime \prime}
$$

and

$$
\omega_{0}=\frac{C_{1}^{\prime}}{B^{\prime}} .
$$

If the correction item is ignored, the chemical potential will be reduced to

$$
\omega_{0}=p_{\theta}\left(\frac{r_{H}^{\prime}}{r_{H}^{2}}-a_{k} \sin \theta_{0}\right)
$$

where $p_{\theta}$ has been defined in Eq. 35 .

\section{CONCLUSION}

Based on the Lorentz symmetric violating theory, the semiclassical theory and the quantum tunneling radiation theory, we get the dynamical equation of Dirac particles by studying Eq. 1, namely the Dirac-Hamilton-Jacobi equation shown as Eq. 14 or Eq. 16. After giving explicit formula of the ether-like field vector $u^{\mu}$ and solving Eq. 16, the corrected tunneling rate of Dirac particles and the corrected Hawking temperature at the event horizon of the Kinnersley black hole are obtained. These new results are of great significance for 
further studying the thermodynamic evolution of black holes. It is necessary to further note that the key to solve Dirac-Hamilton-Jacobi equation is to construct the etherlike field vector correctly. The specific form of $u^{\mu}$ must be selected according to the characteristics of the curved spacetime to be investigated, so as to ensure the validity of the derivation. The reference time $v_{0}$ and the reference angle $\theta_{0}$ in Eq. $\mathbf{2 8}$ are arbitrarily selected, so the results derived from the general tortoise coordinate transformation are of universal significance. In addition, the entropy $S$ of black hole is closely related to Hawking temperature of black hole. Using the change of Bekenstein-Hawking entropy $\Delta S_{\mathrm{BH}}$ to express the tunneling rate will give $\Gamma \sim e^{\Delta S_{B H}}$. Therefore, the entropy of the black hole should also be corrected. If the Lorentz symmetric violating is not considered, the results in this paper will return to the uncorrected cases that have been known ubiquitously.

\section{REFERENCES}

1. Kraus P, Wilczek F. Self-interaction Correction to Black Hole Radiance. Nucl Phys B (1995) 433:403-20. doi:10.1016/0550-3213(94)00411-7

2. Parikh MK, Wilczek F. Hawking Radiation as Tunneling. Phys Rev Lett (2000) 85:5042-5. doi:10.1103/physrevlett.85.5042

3. Hemming S, Keski-Vakkuri E. Hawking Radiation from Ads Black Holes. Phys Rev D (2001) 64:044006. doi:10.1103/physrevd.64.044006

4. Iso S, Umetsu H, Wilczek F. Anomalies, Hawking Radiations, and Regularity in Rotating Black Holes. Phys Rev D (2006) 74:044017. doi:10.1103/ physrevd.74.044017

5. Parikh MK. The Tenth Marcel Grossman Meeting. Singapore: World Scientific (2006). p. 1585-90.

6. Zhang J, Zhao Z. Charged Particles' Tunnelling from the Kerr-Newman Black Hole. Phys Lett B (2006) 638:110-3. doi:10.1016/j.physletb.2006.05.059

7. Akhmedov ET, Akhmedova V, Singleton D. Hawking Temperature in the Tunneling Picture. Phys Lett B (2006) 642:124-8. doi:10.1016/ j.physletb.2006.09.028

8. Srinivasan K, Padmanabhan T. Particle Production and Complex Path Analysis. Phys Rev D (1999) 60:024007. doi:10.1103/physrevd.60.024007

9. Di Criscienzo R, Vanzo L. Fermion Tunneling from Dynamical Horizons. Europhys Lett (2008) 82:60001. doi:10.1209/0295-5075/82/ 60001

10. Lin K, Yang SZ. Fermion Tunneling from Higher-Dimensional Black Holes. Phys Rev D (2009) 79:064035. doi:10.1103/physrevd.79.064035

11. Das A, Banerjee N. Unitarity in Reissner-Nordström Background: Striding Away from Information Loss. Eur Phys J C (2019) 79:475. doi:10.1140/epjc/ s10052-019-6991-1

12. Feng ZW, Ding QC, Yang SZ. Eur Phys J C (2019) 79:455. doi:10.1140/epjc/ s10052-019-6959-1

13. Yang S, Lin K. Modified Fermions Tunneling Radiation from Kerr-Newmande Sitter Black Hole. Sci Sin.-Phys Mech Astron (2019) 49:019503. doi:10.1360/ sspma2018-00307

14. Shankaranarayanan S, Padmanabhan T, Srinivasan K. Hawking Radiation in Different Coordinate Settings: Complex Paths Approach. Class Quan Grav. (2002) 19:2671-87. doi:10.1088/0264-9381/19/10/310

15. Zhao Z, Dai X. Hawking Radiation from a Non-static Black Hole. Chin Phys Lett (1991) 8:548.

16. Kerner R, Mann RB. Fermions Tunnelling from Black Holes. Class Quan Grav. (2008) 25:095014. doi:10.1088/0264-9381/25/9/095014

17. Kerner R, Mann RB. Charged Fermions Tunnelling from Kerr-Newman Black Holes. Phys Lett B (2008) 665:277-83. doi:10.1016/j.physletb.2008.06.012

18. Lin K, Yang S. Fermions Tunneling of Higher-Dimensional Kerr-Anti-De Sitter Black Hole with One Rotational Parameter. Phys Lett B (2009) 674: 127-30. doi:10.1016/j.physletb.2009.02.055

\section{DATA AVAILABILITY STATEMENT}

The original contributions presented in the study are included in the article/Supplementary Material, further inquiries can be directed to the corresponding author.

\section{AUTHOR CONTRIBUTIONS}

Z-EL completed all the derivation and the paper of writing. JZ checked the errors in equations. S-ZY put forward the idea.

\section{FUNDING}

This work is supported by the National Natural Science Foundation of China (No.11273020,U2031121) and Shandong Provincial Natural Science Foundation, China (No.ZR2019MA059).

19. Liu ZE, Tan X, Liu YZ, Sha B, Zhang J, Yang SZ. Lorentz Symmetry Violation and the Tunneling Radiation of Fermions with Spin 1/2 for Kerr Anti-de-sitter Black Hole (2020), arXiv:2002.01148.

20. Zhang J, Liu ZE, Sha B, Tan X, Liu YZ, Yang SZ. Influence of Lorentz Invariation Violation on Arbitrarily Spin Fermions Tunneling Radiation in the Vaidya-bonner Spacetime. Adv High Energ Phys. (2020) 2020:2742091. doi:10.1155/2020/2742091

21. Liu Z-E, Zhang J, Yang S-Z. Lorentz Symmetry Violation and beyond Semiclassical Theory in the Curved Space-Time of the Arbitrarily Dimensional Reissner-Nordström Black Hole. Results Phys (2021) 29: 104710. doi:10.1016/j.rinp.2021.104710

22. Sakalli I, Övgün A. Uninformed Hawking Radiation. EPL (2015) 110:10008. doi:10.1209/0295-5075/110/10008

23. López FM. The Information Loss Paradox (2019). arXiv:1911.13222.

24. Goldberger W, Rothstein IZ. Virtual Hawking Radiation. Phys Rev Lett (2020) 125:211301. doi:10.1103/PhysRevLett.125.211301

25. Mann BR, Murk S, Terno DR. Paradoxes before the Paradox: Setting up the Information Loss Problem (2021). arXiv:2109.13939.

26. Ferreira RZ, Heissenberg C. Super-hawking Radiation. J High Energ Phys (2021) 2021:38. doi:10.1007/jhep02(2021)038

27. Banerjee R, Majhi BR. Quantum Tunneling beyond Semiclassical Approximation. J High Energ Phys. (2008) 2008:095. doi:10.1088/11266708/2008/06/095

28. Banerjee R, Majhi BR. Quantum Tunneling and Back Reaction. Phys Lett B (2008) 662:62-5. doi:10.1016/j.physletb.2008.02.044

29. Banerjee R, Majhi BR, Samanta S. Noncommutative Black Hole Thermodynamics. Phys Rev D (2008) 77:124035. doi:10.1103/ physrevd.77.124035

30. Banerjee R, Majhi BR. Connecting Anomaly and Tunneling Methods for the Hawking Effect through Chirality. Phys Rev D (2009) 79:064024. doi:10.1103/ physrevd.79.064024

31. Modak SK. Corrected Entropy of BTZ Black Hole in Tunneling Approach. Phys Lett B (2009) 671:167-73. doi:10.1016/j.physletb.2008.11.043

32. Majhi BR. Fermion Tunneling beyond Semiclassical Approximation. Phys Rev D (2009) 79:044005. doi:10.1103/physrevd.79.044005

33. Lin K, Yang S-Z. Quantum Tunnelling in Charged Black Holes beyond the Semi-classical Approximation. Europhys Lett (2009) 86:20006. doi:10.1209/ 0295-5075/86/20006

34. Casana R, Cavalcante A, Poulis FP, Santos EB. Exact Schwarzschild-like Solution in a Bumblebee Gravity Model. Phys Rev D (2018) 97:104001. doi:10.1103/PhysRevD.97.104001

35. Kanzi S, Sakallı İ. Gup Modified Hawking Radiation in Bumblebee Gravity. Nucl Phys B (2019) 946:114703. doi:10.1016/j.nuclphysb.2019.114703

36. Övgün A, Jusufi K, Sakallı İ. Exact Traversable Wormhole Solution in Bumblebee Gravity. Phys Rev D (2019) 99:024042. doi:10.1103/ physrevd.99.024042 
37. Chen S, Wang M, Jing J. Polarization Effects in Kerr Black Hole Shadow Due to the Coupling between Photon and Bumblebee Field. J High Energ Phys (2020) 2020:54. doi:10.1007/jhep07(2020)054

38. Jha SK, Rahaman A. Bumblebee Gravity with a Kerr-Sen like Solution and its Shadow. Eur Phys J C (2021) 81:345. doi:10.1140/epjc/s10052-021-09132-6

39. Kanzi S, Sakallı İ. Greybody Radiation and Quasinormal Modes of Kerr-like Black Hole in Bumblebee Gravity Model. Eur Phys J C (2021) 81:501. doi:10.1140/epjc/s10052-021-09299-y

40. Oliveira R, Dantas DM, Almeida CAS. Quasinormal Frequencies for a Black Hole in a Bumblebee Gravity. EPL (2021) 135:10003. doi:10.1209/0295-5075/ac130c

41. Cruz MB, Bezerra de Mello ER, Petrov AY. Thermal Corrections to the Casimir Energy in a Lorentz-Breaking Scalar Field Theory. Mod Phys Lett A (2018) 33:1850115. doi:10.1142/s0217732318501158

42. Nascimento JR, Yu Petrov A, Reyes CM. Lorentz-breaking Theory with Higher Derivatives in Spinor Sector. Phys Rev D (2015) 92:045030. doi:10.1103/ physrevd.92.045030

43. Pu J, Yang S-Z, Lin K. Lorentz-violating Theory and Tunneling Radiation Characteristics of Dirac Particles in Curved Spacetime of Vaidya Black Hole. wlxb (2019) 68:190401. doi:10.7498/aps.68.20190437
44. Kinnersley W. Field of an Arbitrarily Accelerating point Mass. Phys Rev (1969) 186:1335-6. doi:10.1103/physrev.186.1335

Conflict of Interest: The authors declare that the research was conducted in the absence of any commercial or financial relationships that could be construed as a potential conflict of interest.

Publisher's Note: All claims expressed in this article are solely those of the authors and do not necessarily represent those of their affiliated organizations, or those of the publisher, the editors and the reviewers. Any product that may be evaluated in this article, or claim that may be made by its manufacturer, is not guaranteed or endorsed by the publisher.

Copyright $\odot 2021$ Liu, Zhang and Yang. This is an open-access article distributed under the terms of the Creative Commons Attribution License (CC BY). The use, distribution or reproduction in other forums is permitted, provided the original author(s) and the copyright owner(s) are credited and that the original publication in this journal is cited, in accordance with accepted academic practice. No use, distribution or reproduction is permitted which does not comply with these terms. 